\title{
On the design of an urban modelling platform and its application for a New York analysis
}

\author{
Ursula Eicker $^{1,2}$, Juergen Schumacher ${ }^{2}$, Verena Weiler ${ }^{1}$, Reiner Braun ${ }^{3}$ \\ ${ }^{1}$ Center for Sustainable Energy Technology, University of Applied Sciences Stuttgart, Stuttgart, \\ Germany \\ ${ }^{2}$ Gina Cody School of Engineering and Computer Science, Concordia University, Montréal, \\ Canada \\ ${ }^{3}$ University of Applied Sciences Reutlingen, Faculty of Informatics, Herman Hollerith Center \\ (HHZ) Böblingen, Germany
}

\begin{abstract}
Urban platforms are essential for smart and sustainable city planning and operation. Today they are mostly designed to handle and connect large urban data sets from very different domains. Modelling and optimisation functionalities are usually not part of the cities software infrastructure. However, they are considered crucial for transformation scenario development and optimized smart city operation. The work discusses software architecture concepts for such urban platforms and presents case study results on the building sector modelling, including urban data analysis and visualisation. Results from a case study in New York are presented to demonstrate the implementation status.
\end{abstract}

\section{Introduction}

Supporting the planning and operation of smart and sustainable cities with a minimized $\mathrm{CO}_{2}$-footprint is a huge challenge for the emerging urban modelling community. The process involves combining knowledge from different domains. An Urban platform should involve all stakeholders with focused transformation strategies.

Analysing the best transformation strategies for complex urban systems involves modelling of the buildings, supply and distribution systems, transport and logistics. Furthermore, it needs interaction among citizens and local stakeholders for both operations and strategic planning (Musa, 2018). Additionally, applications and processes in smart cities also need to make efficient use of the rapidly growing information and communication (ICT) infrastructure for collecting, processing, and sharing information (ChuanTao, et al., 2015). This requires a reliable communication and networking infrastructure and big data handling as the backbone of smart cities (Pande Rana, 2018). Innovation, e-participation and smart technology applications offer ample scope for citizen engagement (Bolivar \& Munoz, 2019).

Smart city services rely on urban ICT platforms, which offer seamless interconnection with monitoring systems at the infrastructure level. On top, big data structures allow for storing and analysing the generated information, which can eventually be offered to third parties through standardized interfaces in an open data fashion (Vilajosana, et al., 2013). Cluster or Neighbourhood Management Systems can support efficient integration of renewable energy sources into existing energy networks
(Rodriguez, Brennenstuhl, Yadack, Boch, \& Eicker, 2019). The ICT systems need to match user demands and available supply capacity from renewable energy sources while activating storage and demand response. Innovative data analytics methods should be combined with white or grey box models to provide optimum operation and forecasting capacities to the urban platform (Nouvel, Zirak, Coors, \& Eicker, 2017).

Large-scale urban data networks and Internet-of-Things platforms provide real-time data on citizen mobility patterns, air quality, thermal comfort, urban infrastructure performance, and more. Smart metering rollouts are slowly contributing to map the energy consumption patterns within the low voltage network and could be used for large-scale demand side management.

Such city usage data are very much needed for modelling and model calibration efforts of low-carbon urban districts, where the buildings, industrial and transport related energy demand should be supplied by renewable energy systems and matched by demand-side management actions and short to seasonal storage. Information sources can be building registers, census, cadastre records and geospatial datasets. However, to integrate information from different sources the data has to be organized in a structured way and semantic support has to be provided.

The scale and complexity of status-quo modelling of urban districts, the prediction of future development trends of the next decades such as population growth, behaviour and technological changes and the integration of urban big data has resulted in diverse software systems addressing individual features of the urban modelling problem.

\section{Use cases for an urban platform}

\section{Strategies for zero carbon development}

One major use case is to model the status quo carbon balance of a city with all consumption sectors included, validate the model with monitored data and then project scenarios for future development. The modeling approach should be built on three-dimensional urban geometry, including the street layout and land use and should calculate all building's heating, cooling and electricity demand, transport related energy consumption as well as energy consumption and flexibility of urban infrastructure such as water supply, waste water treatment plants and food production, distribution and consumption. 


\section{Urban load forecasting using weather predictions}

Weather forecasting combined with urban microclimate modeling allows to adjust the modeling boundary conditions for solar irradiance, temperature, humidity and wind speed. The building sector energy demand can thus be predicted for a given forecasting time (typically 1 to 3 days), which allows to derive DR strategies with peak shaving or storage charging adapted to available renewable energy supply.

The same modeling toolset can also be used to predict the urban microclimate and urban energy consumption for longer term climate change related weather changes. A relevant use case is to model urban heat island effects and mitigation strategies through greening or evaporative cooling.

\section{Transport related energy consumption, emissions and air quality}

The transport sector as one of the major urban $\mathrm{CO}_{2}$ producers needs to drastically change. The urban platform should allow to map current transport conditions, calculate the energy and $\mathrm{CO}_{2}$ emissions related to transportation and then model scenarios of technological change such as electrification of the transport sector or synthetic fuel use from Power to X systems.

\section{Citizen participation and evaluation of scenarios}

As the involvement of citizens is a core element of a smart city, well designed graphical user interfaces are crucial to involve citizens and urban stakeholders. 3D visualization of mapped data and modeled scenarios are extremely well suited to inform citizens on possible scenarios and have them interact with comments, drawings, suggestions.

\section{Business models for energy efficiency and renewables, smart mobility and other resource sharing concepts}

The urban platform should evaluate business models for renewable integration, for example calculate cost-benefits of participation in electricity markets, but also for providing data services to very different stakeholders and citizens. This might include better risk evaluation for the insurance industry through sensor data information such as water leakage detection, services for customer acquisition by providing heat or cooling demand information or age of equipment or similar.

New financing concepts for the urban transformation

The urban platform has to contain methods to calculate the financial viability of building efficiency scenarios, land use changes or new mobility concepts. This involves data bases for cost functions for the building stock retrofit or for renewable energy systems, cost and estimation of units for different mobility concepts or similar. The platform user should be able to select scenarios and vary assumption related to cost and their development. As an outcome, various financing options should be suggested.

\section{Urban modelling software overview}

Urban modelling environments such as SimStadt from HFT Stuttgart, URBio and CitySim from EPFL Lausanne, KomMod from Fraunhofer ISE, Urban Modelling
Interfaces (UMI) for EnergyPlus dynamic building simulation from MIT Boston, UBEM for ArcGIS from ETH Zurich, EnergyPro from Aalborg University and many more were designed to solve specific urban energy simulation or optimization questions. For example, SimStadt is able to handle 3D CityGML geometry and calculate the urban heating demand or photovoltaic potential in a bottom-up approach, URBio, EnergyPro or KomMod allow to optimize energy system components for a given district load profile, EnergyPlus can be parametrized for a range of building archetypes to scale up to an urban dimension. Tools that optimize the energy system are mostly based on simplified linear component models and do not model distribution networks in detail (for example hydraulic and temperature related losses in district heating systems).

To easily extent the functionality of the existing complex software packages, it would be extremely useful to have a user-friendly graphical workflow editor, which enables the user to specify, extend or combine existing workflow steps or to introduce new models into the urban platform. In SimStadt, workflow steps such as 3D data handling, weather data processing, heating demand simulation, photovoltaic potential calculation or many others are composed as fixed workflows. SimStadt allows only hierarchical workflows, which is a restriction, but they can be parallelized automatically. The main advantage of the workflow engine is that the results are reproducible and that the user can easily parametrize scenarios. The main disadvantage is that the creation of new workflows requires Java programming skills and detailed knowledge of the underlying data model.

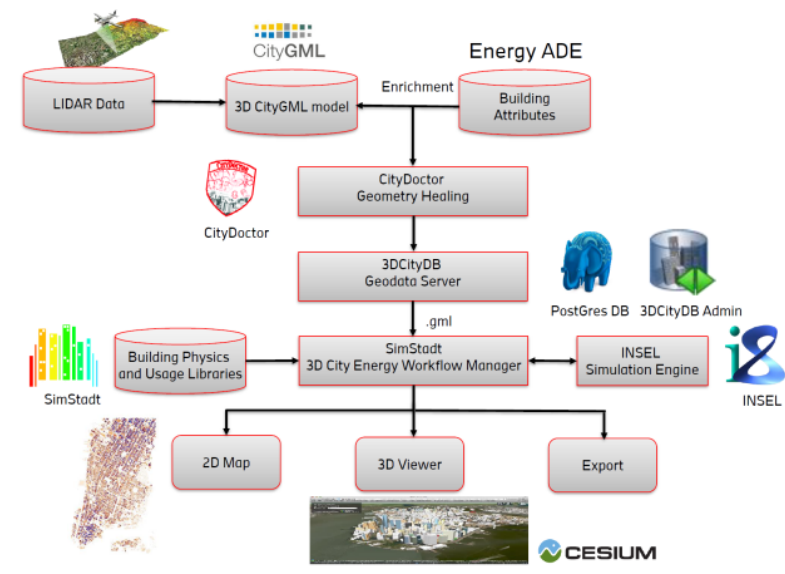

Figure 1: Features of building sector modelling platform part based on work of the SimStadt projects.

\section{Urban software architecture}

An urban platform requires an urban data model with access to heterogeneous data sources and sensors, a data management system, a workflow or web service concept to organize data handling and simulation tasks, and a visualisation engine for user interaction.

So far, urban platforms have mainly focused on urban data management, while not taking into account simulation or optimization tools. The European Innovation Partnership on Smart Cities and Communities 
(EIP SCC) has identified the need to share urban data for social and commercial benefit (see DIN SPEC 91357 on open urban platforms). A lack of a standard to refer cities and suppliers to, led to the design of an overall reference architecture of Open Urban Platforms and related open API characteristics. Such urban platforms specifically focus on the integration of IoT enabled infrastructure and assets in cities, enable re-use and re-purposing of urban data from these sources by combining them with other urban data from other sources, and make these available to applications, smart services, and other management systems.

Cities have different urban infrastructures, which are operated by different infrastructure providers. These infrastructures are largely operated with existing management systems. Examples are building, traffic or street light management systems, electricity grid systems, or water management systems. All in all, a variety of management systems already exist to which an open urban platform shall connect to. Today, these systems are mostly not interconnected with each other and rarely exchange data, which is also due to security reasons. Simulation or optimisation models are not yet part of such urban platforms.

\section{Urban data model}

The urban data management and modelling platform in this work is based on 3D city models described by the OGC Standard CityGML. CityGML is an open data model and stores the city model based on Extensible Markup Language (XML) format (see Figure 2).
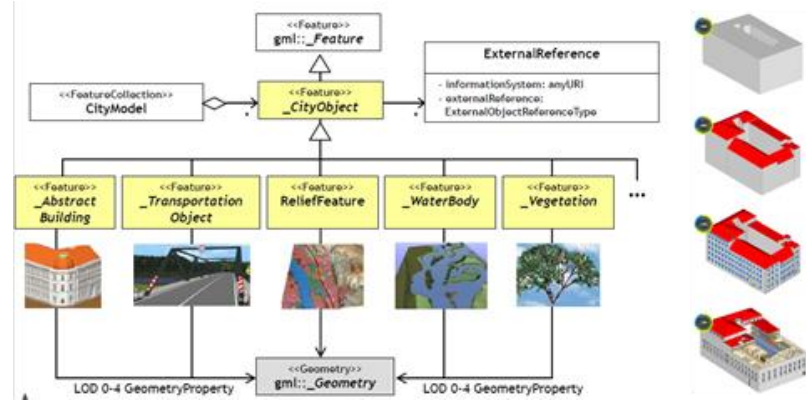

Figure 2: CityGML data structure with four levels of geometry detail shown at the right (Copyright OGC)

CityGML as an application independent city information model has shown a lot of promise within the geospatial domain. A considerable asset of CityGML is its flexibility. This ensures an object in a city can be modelled with different Level of Detail, thereby enabling the virtual city objects to adapt to the scale of local urban surroundings. Furthermore, geospatial data of most cities are now readily available as open data in CityGML compliant virtual 3D city models (see OGC data collection OGC, www.opengeospatial.org/data; or more specifically Berlin 3D-Download Portal, 2015, https://daten.berlin.de/datensaetze/lod2geb\%C3\%A4udedaten-berlin;

3D City Model of New York City, 2015, https://www1.nyc.gov/site/doitt/initiatives/3d-

building.page). With the concept of Application Domain
Extensions (ADE) in CityGML it is also possible to incorporate domain-specific entities. For example, the Utility Network ADE (2017) allows modelling of gas, waste water, district heating/cooling networks and electricity grids with 3D topographic city objects, or the Energy ADE (2017) extends CityGML by a number of energy-related features and attributes in order to perform energy simulations at city scale but with the granularity that reach down to a single building.

\section{Sensor data integration and visualization}

The Urban platform should take advantage and integrate already deployed sensors. However, the heterogeneous sensor systems and irregular time-series data streams from numerous IoT devices make it a difficult task. To address this, the platform must be capable to aggregate or interpolate the data over time. Recently, it was shown that integration of data from heterogeneous sensor systems can be done in an effective and efficient way using the OGC SensorThings API (Santhanavanich et al., 2018). The use case relied on pedelec and user fitness data. Furthermore, open-sources 3D globe (Cesiumjs library) was used to visualize the integrated sensor data.

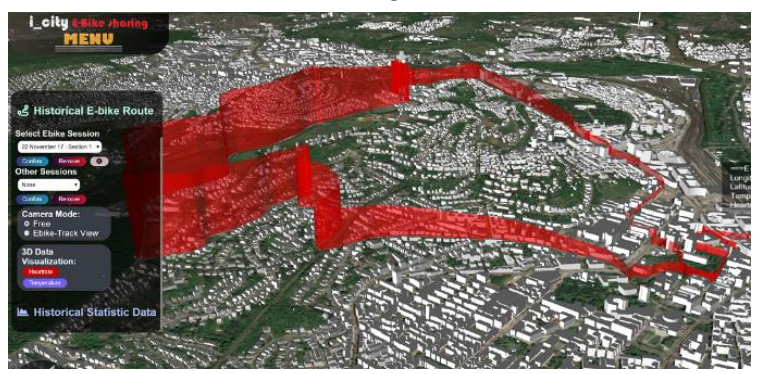

Figure 3: Visualizing of data: heart rate data along the e-bike route in Stuttgart, Germany

\section{Server oriented architecture (SOA)}

A Service-oriented architecture (SOA) exposes software functionalities as Web Services (see Figure 4).

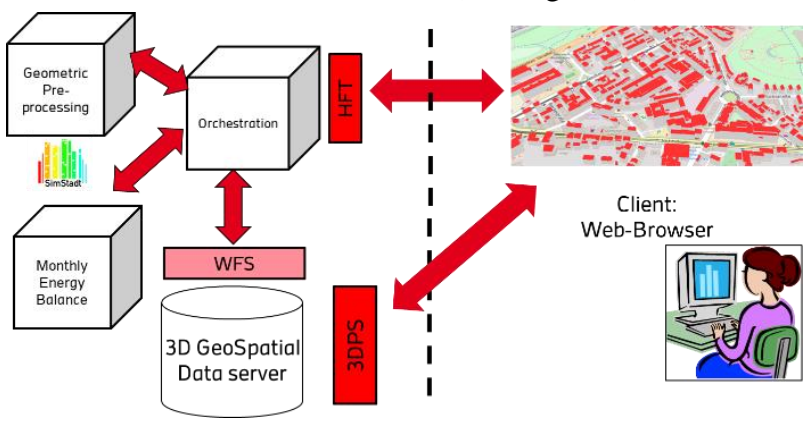

Figure 4: Software as a service concept 
SOA is made up of three core entities (see Figure 5).

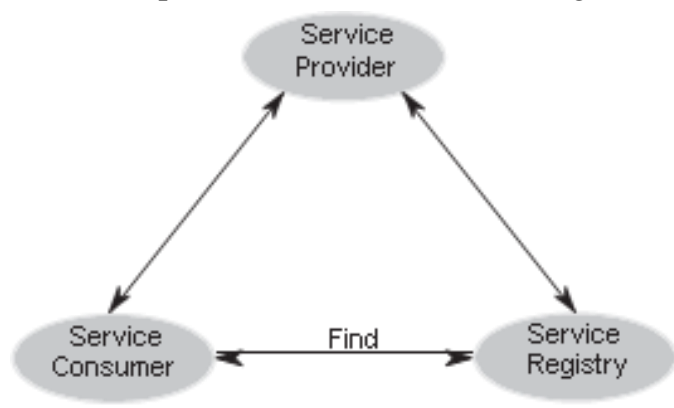

Figure 5: Service oriented architecture entities

The SERVICE PROVIDER is an entity that provides a software functionality. The SERVICE CONSUMER is an entity that uses the software functionality. The SERVICE REGISTRY is an entity that enables the SERVICE PROVIDER to publish its software functionality and allows the SERVICE CONSUMER to search for a software functionality that it requires to fulfil a task. Each of these entities relies on key standardized technologies (i.e., SOAP, WSDL, UDDI). Simple Object Access Protocol (SOAP) (Gudgin, et al., 2007) defines a messaging standard based on Extensible Markup Language (XML) and, with the help of the Hypertext Transfer Protocol (HTTP), provides a communication protocol for accessing Web Services. The Web Service Description Language (WSDL) (Christensen, Curbera, Meredith, \& Weerawarana, 2001) is used to describe the web service access interface. Universal Discovery, Description and Integration (UDDI) (Clement, Hately, von Riegen, \& Rogers, 2004) is a registry that allows advertisement and discovery of web services thereby providing the opportunity to dynamically bind a Web Service at runtime.

Within the proposed Urban Data Platform concept, streams of data are collected in a PostgreSQL database and provided to the user as a service. For example, to provide the service of delivering 3D building data, a request uniform resource identifier (URI) is created with a specific layer name to the 3D Portrayal Service. Other relevant information is added including the service name, the version, the request, the format, the coordinate system information, and the bounding box positions.

\section{Workflow management and simulation engine}

The Web service orchestration of different geoinformation and modelling tasks faces the difficulty of result reproducibility, as each of the distributed services can be changed locally and it is a challenge to keep version control of the functionality of each service.

A more controlled alternative to the SOA architecture are scientific workflows (Curcin \& Ghanem, 2008). They are able to (a) integrate large data sets of different kind and at different level, e.g. building geometries and features, heating networks, patterns of energy consumption, production and conversion, (b) third-party simulation systems, and (c) inhomogeneous hardware resources like database servers, simulation engines, web services, graphics cards and workstations. Moreover, they can provide a modern graphical user interface and enable fast parallel computation. However, no standard for such scientific workflow systems exists yet. To avoid locking into a special software product, we decided to build upon Java and its rich ecosystem. Especially, new language idioms of Java 8 provide features common to scientific workflow systems like high-level support for parallel computing, domain specific languages, functional programming, and generic user interfaces. Last but not least, Java provides industry-proven support for XML processing that comes very handy for the kind of data we have to deal with in urban modelling. The modelling platform SimStadt is based on this Java based scientific workflow system.

SimStadt utilizes the library citygml4 $\mathrm{j}$ to load, process and store CityGML files with Java. This library provides a Java API and Java classes for all standard CityGML types so that a CityGML model can be instantiated in Java applications. Furthermore, citygml4j provides a JAXBXJC binding compiler (ade-xjc) which conveniently generates Java classes from custom ADE XML schema definitions like the Energy ADE, so that the newly introduced energy-related attributes and types are now able to be loaded and stored together with a standard CityGML model by SimStadt.

The structure of computational tasks can be best described as hierarchical workflows. Such workflows basically consist of chains of processing steps that create, transform and consume specific data objects step by step. Conforming to the disaggregation of model objects from high-level to low-level (e.g. city > district $>$ building > building zone $>$ wall) often a processing step at a higher level is realized by a chain of steps working on lowerlevel model objects, thus the term "hierarchical". In some cases, chains of processing steps at one level may be logically independent, and thus be executed concurrently. Note, that in this schema, circular processing of steps is not allowed.

While dealing with large-scale models based on different data sources, the risk of errors and incoherent results are generally very high. A Graphical User Interface (GUI) enables to navigate in the different workflows and workflow steps, allowing for the analysis of the intermediary results at each step of the workflow, through charts, tables and other output. The GUI also enables the user to modify the hypotheses and parameters of workflow steps and create scenarios accordingly.

To construct and validate urban data handling and modeling applications, a graphical editor or workbench system is extremely useful to design and test models before finally implementing them in a workflow management or web service orchestration system. The urban platform designed by the authors so far uses a custom designed graphical framework named Vseit, programmed in Java and communicating with the INSEL simulation engine for simulation model execution. INSEL is a simulation environment with a domain specific graphical programming language that flexibly allows to program simulation models using a wide range of block libraries. The main functionalities in INSEL are from the 
domain of renewable energy systems, building modeling and meteorology, but can be easily extended by user blocks.

The programming concept of INSEL also allows to use the simulation engine to sort and execute third party simulation models or data access, so that INSEL can be used for the orchestration of urban simulation models

\section{Case study application}

The modelling approach was applied to a case study in the New York City district Borough Hall (see Figure 6), where SimStadt was used to simulate all the buildings in the district connected to a single substation with hourly resolved electricity monitoring data.

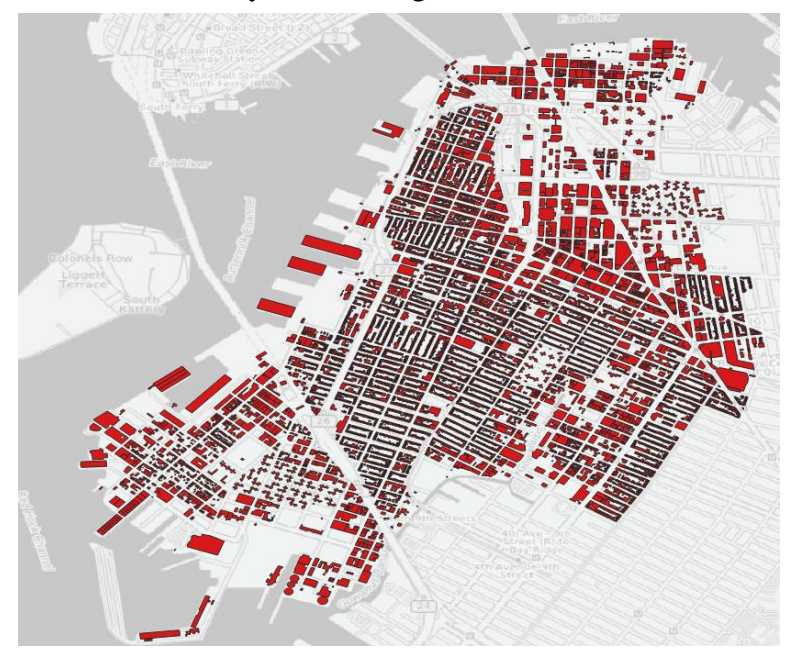

Figure 6: Buildings in the case study area in Brooklyn, $N Y C$

In addition to the building and supply system energy consumption, the energy demand of further sectors such as food supply, distribution and preparation, waste water treatment, and transport was analysed to get a full picture of the status quo consumption and then derive scenarios towards a zero-carbon district. Data related to the energy demand of the building, food and water sector was collected from various sources and prepared for analysis.

The goal of the City of New York is to reduce the greenhouse gas emissions by $80 \%$ until 2050 (Technical Working Group, The City of New York). To achieve this goal, a main focus is on buildings and their heating, cooling and electricity demand.

\section{Data sources}

All data used are open data. The relevant data are including energy usage data (provided by Consolidated Edison, Inc.), land use and geographic data at the tax lot level (MapPluto, provided by Department of City Planning, NYC), data including demographics, financial conditions and heating systems at the census tract level (provided by U.S. Census Bureau), building footprint and points of interest from OpenStreetMap (provided by Geofabrik $\mathrm{GmbH}$ ), and other data are from NYC Open Data (https://opendata.cityofnewyork.us). The threedimensional (3D) building massing model of New York City is provided online from DOITT in the CityGML format (https://www1.nyc.gov/site/doitt/ initiatives/3d- building.page). This dataset is later on converted to $3 \mathrm{D}$ Tile format as a service with the OGC 3D Portrayal Service standard implemented by the Fraunhofer Institute (Reference: OGC Testbed 13 ER). This service allows users and developers to request and interact online with the $3 \mathrm{D}$ building dataset.

The overall system architecture for this case study is shown in Figure 7.

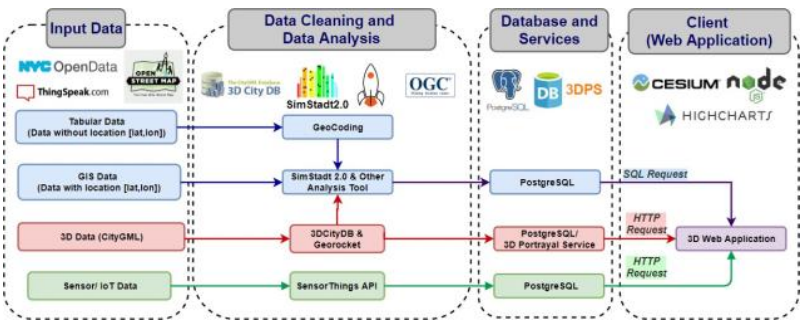

Figure 7: System architecture for data analysis and visualisation

The tabular data provided by NYC OpenData and the U.S Census Bureau has no geospatial coordinate provided. Therefore, to process the data with other layers of data, the geocoding from address to geospatial location had been processed in Python using the NYC geocoding service

(https://developer.cityofnewyork.us/api/geoclient-api). Complementing data not available on lot level (mostly census tract data) was superimposed on the combined datasets. Then, the output data was integrated and analysed with other layers of data in both SimStadt and the QGIS platform.

The 3D data in CityGML format (shown in red colour in Figure 7) is imported into a 3D City Database. It is an open-source 3D geo-database to store, represent, and manage virtual 3D city models on top of a standard spatial relational database PostgreSQL with PostGIS. Examples are the 3DcityDB from TU Munich or Georocket from Fraunhofer IGD.

Next, sensor and temporal data (shown in green colour) can be imported to the database following the OGC SensorThings API standard. The OGC SensorThings API is an OGC community standard providing an open and unified framework to interconnect IoT devices, data, and applications. It allows the IoT devices and applications to create, read, update, and delete IoT data and metadata through a HTTP request (http://docs.opengeospatial.org/is/15-078r6/15-

078r6.html). Other calculated data or analysed data are imported to the database by using the Node.js framework which has an advantage for coding in the same language on both server side and client side. As all the input data are from the heterogeneous sensor systems, data integration is a major challenge. In this work, several standards are used together to tackle this task.

\section{Modelling results and visualisation}

\section{Results of data analysis and energy simulations}

The first analysis is to assess the status quo of the district under investigation. The aforementioned data sources 
were analysed to find useful information concerning energy demand or energy systems and infrastructure.

Figure 8 shows the share of fuel types used for heating in each of the census tracts inside the Borough Hall case study district as pie charts. In most of the tracts, utility gas is dominating, however there are some tracts that have a significant share of electrical heating, a few are even dominated by oil. Another important information is the type of heating system in the buildings, e.g. if it is a central or decentral system, which can partly be deduced from the fuel type. It is more probable that buildings with electrical resistance heating install heat pumps fed by renewable electricity compared to an existing central heating system with gas. Electrical heating is predominant in newer office buildings in Downtown Brooklyn (upper right part of Figure 8. Also visible in figure 8 is the year built of the buildings in blue and the ratio of house owners in each census tract district (green).

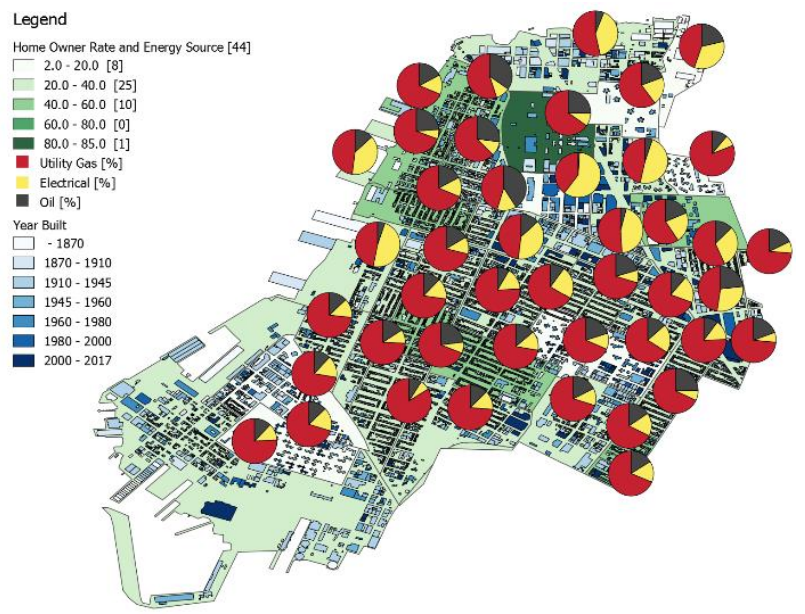

Figure 8: Home owner rate, energy source, fuel type distribution and year built in Borough Hall

Different strategies to stimulate the desired modernisations could be applied, depending on this information. Districts with a high ratio of home ownership and high income population can afford the needed modernisations more easily while in other districts with low income or city owned apartments other stimuli are needed. Also, considering the building age, a priority between building refurbishment and system modernisation can be determined.

Finding these different indicators and combining them can help to identify high-impact quarters for immediate or future modernisation leading to a more realistic roadmap for the ambitious goals regarding greenhouse gas emission reduction of the City of New York.

The heating and cooling demand of the whole district is simulated with SimStadt.

One scenario to reduce the energy demand in the district is to increase the cooling set point by $6{ }^{\circ} \mathrm{C}\left(11^{\circ} \mathrm{F}\right)$ from $20{ }^{\circ} \mathrm{C}$ to $26{ }^{\circ} \mathrm{C}$ and the reduction of infiltration losses through unsealed windows and doors. The combination of those two measures results in a reduction of the heating demand by $19 \%$ and a reduction of the cooling demand by $56 \%$, as can be seen in Figure 9. Especially in non- residential buildings with a large cooling load, this could mean a big $\mathrm{CO}_{2}$-reduction potential.

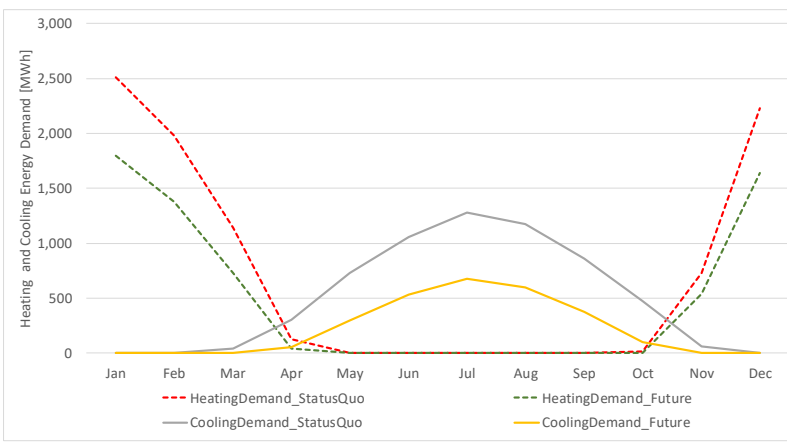

Figure 9: Influence of cooling set point temperature and air tightness on heating and cooling demand

\section{Food sector energy demand}

To meet the goals stated by the government of New York City it is crucial to consider not only the built environment, but also material flows throughout it. Since food is an energy-intensive material flow in cities, the food sector contains potential to reduce both energy demand and resulting $\mathrm{CO}_{2}$-emissions. A stakeholder analysis was conducted and the food supply chain of New York City was mapped to show how market participants are linked and where and how much energy is consumed. These methods are also applicable to a larger district or even the national level.

One objective is to evaluate the current electricity consumption of the food sector. Furthermore, the last mile transportation and the energy potential of the waste generated by the residents and businesses is investigated.

Open data from the above-mentioned sources are used to locate the food related usages (food sales, e.g. supermarkets and food services, e.g. restaurants) and to determine the size of each location to calculate the electrical energy consumption (see Figure 10). This results in an electricity demand for food sector related refrigeration in Borough Hall of $70 \mathrm{GWh} / \mathrm{a}$. With a total electricity consumption of $1.33 \mathrm{TWh} / \mathrm{a}$ (2013), this equals $5.2 \%$ ( $1 \%$ food sales, $4.2 \%$ food services) of the whole electricity demand of the district.

Additional to production and storage, the transportation of food and the resulting energy demand needs to be considered. Therefore, the delivery related emissions from six different distribution points (Hunts Points, College Point, Long Island City, Maspeth, Greenpoint) into the Borough Hall district were analyzed.

The average distance between the distribution centers and the Borough Hall district is $14 \mathrm{~km}$ for both ways and $124,000 \mathrm{kt}$ of food must be transported this distance every year (New York City Economic Development Corporation, NYC Mayor's Office of Recovery \& Resiliency, \#ONENYC, 2016). Assuming that one truck needs 2.7 MJ energy for the transportation of one metric ton of cargo per kilometer, the transportation of food to Borough Hall consumes 2.6 GWh and emits 6,451 kt of $\mathrm{CO}_{2}$ annually. 


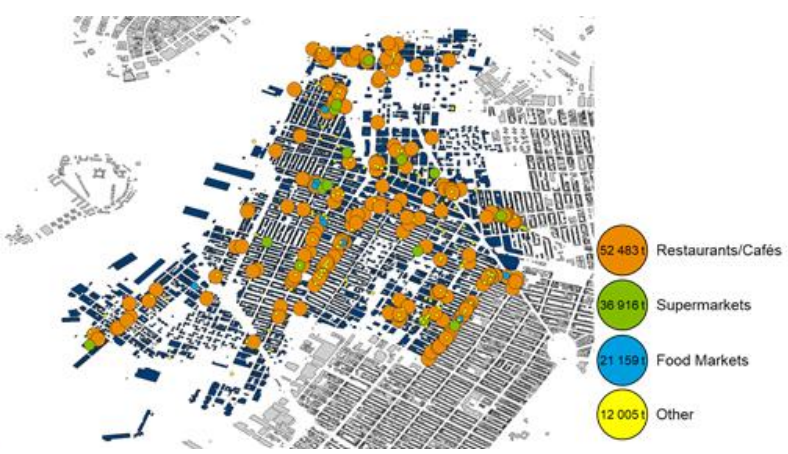

Figure 10: Location of food sales and food services in case study area

\section{Visualization}

The 3D web application of SimStadt is able to visualize the $3 \mathrm{D}$ buildings with their simulated performance. This allows users to see the heating demand or energy use intensity (EUI) immediately according to the building size and use. Moreover, users can visualize and compare different scenarios (e.g. status quo and refurbishments) on the selected value in the selected area with an interactive graph using the Highchart.js library.

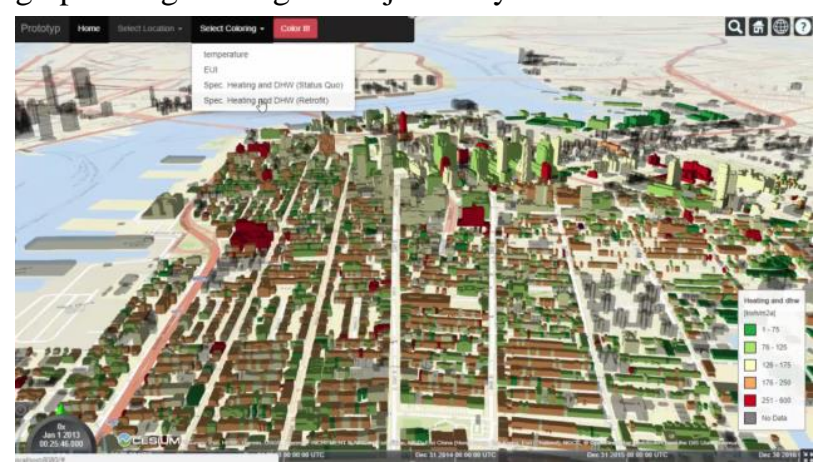

Figure 11: Visualisation of heating demand scenario simulation

The data for the Energy Usage Intensity of large buildings is collected for the years of 2012, 2013, 2014, 2015 and 2016 from NYC OpenData. According to the Local Law 84 , all private buildings over $50,000 \mathrm{ft}^{2}$ and city-owned buildings with over $10,000 \mathrm{ft}^{2}$ have to report their annual energy usage. Utilizing the Cesium Time-scale, the timeseries dataset can be displayed depending on the year it portrays. Cesium grants the possibility to change the speed with which the time moves on the timescale. The speed is set to a few days per second to create an adequate rate for multiple years of data.

If the year of the internal time changes, an event is called that colours the buildings depending on the current year's data.

The data for each year is stored in a PostgreSQL Database. This data is queried from the node.js server. It returns lists of building IDs with their respective EUI. This list is sorted by the predefined scale $\left(\mathrm{kBtu} / \mathrm{ft}^{2}\right)$ into six specific groups.

For displaying the simulation results of specific heating and domestic hot water demand from SimStadt, a different approach was chosen. The status quo and refurbishment scenario are calculated by using the 3D
City GML model and different values for the building physics of the building, such as U-values and infiltration. The calculated output files are then loaded into a PostgreSQL Database. This process provides the advantage of being able to make different requests instead of reading it from the file.

\section{Conclusion}

The paper discusses concepts and implementations of urban data management, modelling and visualization.

An urban platform is proposed that combines 3D geospatial information handling, the integration of metering and sensor data and modelling functionalities. Standards developed by the Open Geospatial Consortium (OGC) are the basis of the data model and 3D processing services.

The use of multiple data sources can be challenging, because they are not always on the same level and scale and are usually in different data formats. Combining these diverse data sources however provides information that can be crucial to making sensible scenarios for a future renewable and $\mathrm{CO}_{2}$-reduced city planning.

Two software architecture concepts have been implemented and tested: a software as a service concept with distributed web services and a desktop workflow management system. The Workflow management system was used in a case study to analyse the status quo and scenarios for an urban district in Brooklyn, New York. It could be shown that in the building sector $45 \%$ of carbon savings can be achieved by refurbishing the entire building stock from status quo to a low energy standard. Increasing the cooling setpoint temperature by $6 \mathrm{~K}$ and improving the air tightness alone allows to reduce the district cooling energy by $56 \%$ and the heating energy by $19 \%$. Using the geoinformation system to locate food related service and sales point allowed to calculate the energy demand for food refrigeration and food logistics. The $70 \mathrm{GWh}$ required correspond to $5.2 \%$ of the whole electricity demand of the district (1\% food sales, $4.2 \%$ food services).

Transformation strategies can thus be based on socioeconomic indicators that help to identify high-impact quarters for immediate or future modernisation leading to a more realistic roadmap for the ambitious greenhouse gas emission reduction goals of New York. City

Web 3D technologies can support a multi-participatory policy in urban planning and are suitable for handling the spatial and temporal data diversity in urban areas. Employing OGC's 3D Portrayal Service in conjunction with Cesium.js and 3D Tiles has proven to be an effective strategy due its flexibility and adaptability.

\section{Acknowledgements}

The work is largely based on a close collaboration between the Research Center for Sustainable Energy Technologies and the Center for Geodesy and Geoinformatics of HFT Stuttgart. Many contributors added valuable input to the paper, amongst them a group of German and New York students at a summer school financed by the Baden Wuerttemberg Foundation. Special thanks are due to contributing HFT researchers Joe 
Santhanavanich, Preston Rodrigues, Eric Duminil, KaiHolger Brassel and Prof. Dr. Volker Coors.

The software concept development is part of the International Energy Agency Task on Net Zero Energy Resilient Public Communities EBC Annex 73.

\section{References}

Bolivar, M., \& Munoz, L. (2019). E-Participation in Smart Cities: Technologies and Models of Governance for Citizen Engagement. Springer Press, Series Public Administration and Information Technology.

Christensen, E., Curbera, F., Meredith, G., \& Weerawarana, S. (2001). Web Services Description Language (WSDL) 1.1 . W3C.http://www.w3.org/TR/2001/NOTE-wsdl20010315.

ChuanTao, Y., Zhang, X., Hui, C., Jing Yuan, W., Daven, C., \& Bertrand, D. (2015, 10). A literature survey on smart cities. SCIENCE CHINA, Information Sciences.

Clement, L., Hately, A., von Riegen, C., \& Rogers, T. (2004). Universal Description Discovery \& Integration (UDDI) version 3.0.2. OASIS. http://uddi.org/pubs/uddi-v3.0.2-20041019.htm.

Curcin, V., \& Ghanem, M. (2008). Scientific workflow systems - can one size fit all? Biomedical Engineering Conference CIBEC. Cairo.

Gudgin, M., Hadley, M., Mendelsohn, N., Moreau, J., Karmarkar, A., \& Lafon, Y. (2007). Simple Object Access Protocol (SOAP) version 1.2 part 0: Primer, second ed. W3C.http://www. w3.org/tr/2007/recsoap12-part0-20070427/.
Musa, S. (2018). Smart cities-A road map for development. IEEE Potentials, DOI: 10.1109/MPOT.2016.2566099.

Nouvel, R., Zirak, M., Coors, V., \& Eicker, U. (2017). The influence of data quality on urban heating demand modeling using 3D city models. Computers, Environment and Urban Systems, pp. 68-80.

Pande Rana, D. (2018, 08). Smart cities: issues and challenges in urban infrastructure. Global Journal for research analysis.

Rodriguez, L., Brennenstuhl, M., Yadack, M., Boch, P., \& Eicker, U. (2019). Heuristic Optimization of Clusters of Heat Pumps: A Simulation and Case Study of Residential Frequency Reserve. Applied Energy, pp. 943-958.

Santhanavanich, T. (2018). Visualization and Analysis of E-bike Usage in $3 D$ City Model by Integration of Heterogeneous Sensor Data. Master's thesis, University of Applied Sciences Stuttgart, Stuttgart, Germany.

Santhanavanich, T., Rodrigues, P., Silberer, J., Loidl, V., \& Coors, V. (n.d.). Vision Zero: a 3D interactive web application for safe routing. Unpublished manuscript.

Technical Working Group, The City of New York. (n.d.). One City Built to Last - Transforming New York City buildings for a low-carbon future.

Vilajosana, I., Llosa, J., Martinez, B., Domingo-Prietoo, M., Angles, A., \& Vilajosana, X. (2013). Bootstrapping Smart Cities through a Self-Sustainable Model Based on Big Data Flows. IEEE Communications Magazine, pp. 128-134. 